\title{
分级结构碳纳米笼高效催化苄胺氧化偶联制 $N$-苄烯丁胺
}

\author{
曾誉吕品蔡跃进高福杰卓欧吴强* \\ 杨立军王喜章* 胡征 \\ (南京大学化学化工学院 介观化学教育部重点实验室 南京 210023)
}

\begin{abstract}
摘要 苄胺氧化偶联制 $N$-芐烯丁胺通常需使用贵金属催化剂, 开发廉价催化剂具有重要研究价值. 本工作以具有大比 表面积和丰富表面缺陷的分级结构碳纳米笼( $\mathrm{hCNCs}$ )作为无金属催化剂, 在无溶剂、 $100{ }^{\circ} \mathrm{C}$ 和常压 $\mathrm{O}_{2}$ 条件下即可实现 芐胺到 $N$-芐烯丁胺的高效转化, 反应 $8 \mathrm{~h}$ 的芐胺转化率和 $N$-芐烯丁胺选择性均可达 $98 \%$, 远优于碳纳米管、还原氧化 石墨烯、活性炭等典型碳材料. hCNC700 样品循环使用 6 次后催化性能基本无衰减，且具有优秀的底物拓展性. hCNC700 的优异催化性能源于其超高的比表面积可提供大量的缺陷活性位点, 而独特的分级孔结构十分有利于反应过 程中的传质，使丰富的表面活性位点(缺陷)得以充分利用.
\end{abstract}

关键词 分级结构碳纳米笼; 无金属催化剂; 缺陷催化; 芐胺; $N$-芐烯丁胺

\section{Hierarchical Carbon Nanocages as Efficient Catalysts for Oxidative Coupling of Benzylamine to $N$-Benzylidene Benzylamine}

\author{
Yu Zeng \\ Pin Lyu Yuejin Cai Fujie Gao \\ Lijun Yang \\ Xizhang Wang* \\ Ou Zhuo \\ Qiang $\mathrm{Wu}^{*}$ \\ (Key Laboratory of Mesoscopic Chemistry of MOE, School of Chemistry and Chemical Engineering, \\ Nanjing University, Nanjing 210023, China)
}

\begin{abstract}
N$-Benzylidene benzylamine is an important pharmaceutical intermediate and industrial chemical. Traditionally, it is synthesized via the reaction between benzylamine and benzaldehyde by using Lewis acid catalysts, which usually suffers from the difficulty of product separation and environmental unfriendliness. An alternative approach is the oxidative coupling of benzylamine catalyzed by metal-based catalysts, which is also beset by the contamination of metallic impurities. Thus, the development of green, metal-free and reusable heterogeneous catalysts is highly attractive. Herein, we report an efficient metal-free catalyst, hierarchical carbon nanocages (hCNCs) synthesized by the in-situ magnesium oxide template method with benzene precursor at different temperatures $\left(700,800\right.$ and $900{ }^{\circ} \mathrm{C}$ ), for the oxidative coupling of benzylamine to $\mathrm{N}$-benzylidene benzylamine. The hCNCs feature multi-scale hierarchical pore structure, high specific surface area and abundant surface defects. The hCNC700 exhibits excellent catalytic performance for the solvent-free oxidative coupling of benzylamine to $\mathrm{N}$-benzylidene benzylamine under mild conditions $\left(100{ }^{\circ} \mathrm{C}\right.$, atmospheric $\left.\mathrm{O}_{2}\right)$. Specifically, after reacting for $8 \mathrm{~h}$, both benzylamine conversion and $N$-benzylidene benzylamine selectivity are larger than $98 \%$, far better than the counterparts of carbon nanotubes, reduced graphene oxide and activated carbon, as well as the reported mesoporous carbon and graphene oxide. The hCNC700 also presents high mass activity, significantly better than the reported carbon-based catalysts. Its catalytic performance is almost unattenuated after 6 times of recycling, exhibiting good stability. By comparison experiments, the catalytic activity of hCNCs results from the intrinsic defects of carbon, and the excellent performance of hCNC700 is mainly attributed to the following aspects: (i) its ultra-high specific surface area can provide abundant surface active sites (defects), (ii) the unique hierarchical pore structure is very conducive to mass transfer in the reaction process, enabling the full utilization of these active sites. In addition, hCNC700 shows good catalytic performance for different substrates, with high catalytic activity ( $>90 \%$ conversion rate for $12 \mathrm{~h}$ reaction) and high selectivity $(>95 \%)$ for oxidative coupling of aromatic methylene amines. This study provides a new avenue for the development of cheap and efficient carbon-based metal-free catalysts. Keywords hierarchical carbon nanocage; metal-free catalyst; defect catalysis; benzylamine; $N$-benzylidene benzylamine
\end{abstract}

\section{1 引言}

亚胺是重要的化工原料及生物医药中间体, 通常采
用醛与胺缩合、伯胺偶联、醇与胺交叉偶联等方法合 成 ${ }^{[1-3]}$. 以 $N$-苄烯丁胺为例, 其主要的制备途径是: (1)通

\footnotetext{
*E-mail: wqchem@nju.edu.cn; wangxzh@nju.edu.cn; Tel.: 0086-025-89681910

Received November 17, 2020; published February 1, 2021

Supporting information for this article is available free of charge via the Internet at http://sioc-journal.cn.

Project supported by the National Key Research and Development Program of China (Nos. 2018YFA0209100, 2017YFA0206500) and the National Natural Science Foundation of China (Nos. 21773111, 21972061, 21832003, 52071174).

项目受国家重点研发计划(Nos. 2018YFA0209100, 2017YFA0206500)和国家自然科学基金(Nos. 21773111, 21972061, 21832003, 52071174)资助.
} 
过苄胺与苯甲醛的缩合反应, 使用 Lewis 酸催化剂, 但 产物难以分离, 不够环境友好 ${ }^{[4]}$; (2)通过茮胺氧化偶联, 使用贵金属或其合金(如 $\mathrm{Pd}^{[5]} 、 \mathrm{Pt} / \mathrm{Sn}^{[6]} 、 \mathrm{Ag}^{[7]} 、 \mathrm{Au}^{[8]}$ 等) 催化剂, 但成本高, 产物残留金属杂质. 针对这些不足, 人们致力于开发高效的非贵金属甚至无金属催化剂.

碳材料具有来源广泛、形态结构丰富、比表面积和 表面性质易调控、物理化学性质稳定等优点, 是一类十

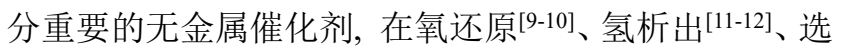
择性氧化 ${ }^{[13-16]}$ 等反应中表现出优良的催化性能. 近年 来, 碳材料在苄胺催化选择氧化制 $N$-茮烯丁胺反应中 展现出诱人的潜力 ${ }^{[17-20]}, \mathrm{Su}$ 等 ${ }^{[17]}$ 报道的碱-酸处理氧化 石墨烯在无溶剂 $90{ }^{\circ} \mathrm{C}$ 条件下可实现 $100 \%$ 的转化率和 $98 \%$ 的选择性. 研究表明, 碳材料的催化活性主要来自 $\mathrm{sp}^{2}$ 碳的本征缺陷(通常指破坏碳六元环结构连续性的缺 陷)、掺杂原子(如 $N 、 B 、 S$ 等)及其 $\pi$ 电子的活化等因 素[21-25].

近年来, 我们课题组采用原位氧化镁模板法开发出 一类分级结构碳基纳米笼新材料, 具有比表面积高、微 孔-介孔-大孔连通共存、表面缺陷丰富和易于掺杂等特 点, 成为开发先进能量转换与存储功能的新平台 ${ }^{[23,26-31]}$. 本工作以分级结构碳纳米笼( $\mathrm{hCNCs}$ ) 作为茮胺氧化偶联 制 $N$-茮烯丁胺反应的无金属催化剂, 无需对其进行任 何修饰，即可获得高的转化率 $(>98 \%)$ 和选择性 $(>98 \%)$, 且具有优良的循环稳定性和底物拓展性. 相 对于碳纳米管、还原氧化石墨烯、商业活性炭等其他碳 材料, hCNCs 独特的分级孔结构十分有利于反应过程中 的传质, 使丰富的表面活性位点(主要是顶角五元环缺 陷、边缘缺陷和孔缺陷三种本征缺陷)得以充分利用 ${ }^{[23]}$, 从而展现出更为优异的无金属催化性能.

\section{2 结果与讨论}

采用原位氧化镁模板法制备 hCNCs, 在 700、800 和 $900{ }^{\circ} \mathrm{C}$ 时制得的样品分别命名为 hCNC700、 $\mathrm{hCNC} 800$ 和 hCNC900. 图 1 是 hCNCs 的扫描电子显微 镜(SEM)和透射电子显微镜(TEM)表征结果. hCNCs 呈 现出由许多纳米片构成的花族状分级结构 (图 1a). 纳米 片是由尺寸约为 $10 \sim 50 \mathrm{~nm}$ 的纳米笼相互连接而成, 纳 米笼的笼壁由一定石墨化程度的不完全连续的碳层构 成, 表明碳纳米笼表面富含缺陷. 随制备温度提高, 碳 层石墨化程度提高、缺陷减少, 且笼壁的石墨化碳层层 数增加(图 1b 1d). 同时, 氧化镁颗粒的尺寸也随制备 温度升高而增大, 使 $\mathrm{hCNCs}$ 的笼腔变大, 比表面减小, 总的缺陷数量也随之减少.

Raman 光谱是表征碳材料石墨化程度及缺陷的有 效手段. 对于 $\mathrm{sp}^{2}$ 碳材料, $1350 \mathrm{~cm}^{-1}$ 附近的 D 带源自缺 陷、无序结构、边角等, $1580 \mathrm{~cm}^{-1}$ 附近的 $\mathrm{G}$ 带源自石墨 化结构. 一般而言, D 带和 $\mathrm{G}$ 带的峰面积比 $\left(I_{\mathrm{D}} / I_{\mathrm{G}}\right)$ 越大, 表示碳材料的石墨化程度越低、缺陷越丰富 ${ }^{[23,32-34]}$. D 带
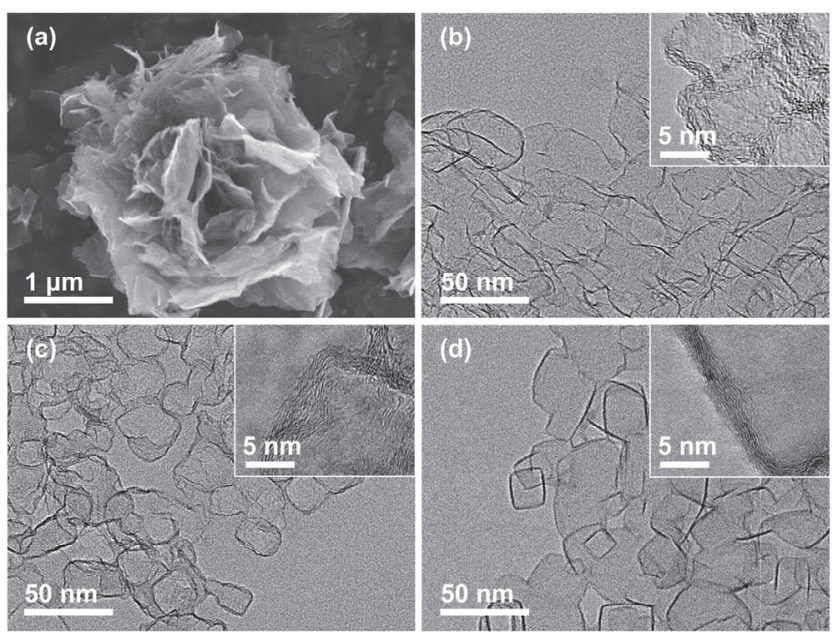

图 $1 \mathrm{hCNCs}$ 的 SEM 及 TEM 表征. (a)典型的 SEM 照片; $(\mathrm{b} \sim \mathrm{d})$ 分别 为 $\mathrm{hCNC} 700 、 \mathrm{hCNC} 800$ 和 $\mathrm{hCNC} 900$ 的 $\mathrm{TEM}$ 照片. 插图为高分辨 TEM 照片.

Figure 1 SEM and TEM characterizations of hCNCs. (a) Typical SEM image; (b d ) TEM images of hCNC700, hCNC800 and hCNC900, respectively. Insets are high-resolution TEM images.

的半峰宽 $(\mathrm{FWHM})$ 与缺陷数量存在正相关关系, 在超低 缺陷的单层石墨烯中 $\mathrm{FWHM}(\mathrm{D})$ 仅约 $20 \mathrm{~cm}^{-1[34]}$, 当缺 陷增加时, FWHM(D) 随之增加 ${ }^{[35]}$. 由 $\mathrm{G}$ 带的半峰宽, 通 过公式 $F W H M(G)=14+430 / L_{a}$ 可以估算出石墨态碳的 平均晶化尺寸 $\left(L_{\mathrm{a}}, \mathrm{nm}\right)^{[36]} ; \mathrm{FWHM}(\mathrm{G})$ 越小, $L_{\mathrm{a}}$ 越大，即碳 材料的石墨化程度和有序度越高. 图 2 是不同温度下制 备和退火处理后得到的 $\mathrm{hCNCs}$ 的 Raman 光谱及其 $I_{\mathrm{D}} / I_{\mathrm{G}}$ 、 $\mathrm{D}$ 带和 $\mathrm{G}$ 带的半峰宽与温度的变化关系图. 随制备温度 从 $700{ }^{\circ} \mathrm{C}$ 升高到 800 和 $900{ }^{\circ} \mathrm{C}, \mathrm{hCNCs}$ 的 $I_{\mathrm{D}} / I_{\mathrm{G}}$ 从 3.1 下降到 2.9 和 $2.7, \mathrm{FWHM}(\mathrm{D})$ 从 $271 \mathrm{~cm}^{-1}$ 减小到 269 和 $249 \mathrm{~cm}^{-1}$, 而 FWHM(G)基本保持在约 $112 \mathrm{~cm}^{-1}$ (图 2b, $2 \mathrm{c})$. 可见，随制备温度升高，碳纳米笼的缺陷减少，与 图 $1 \mathrm{~b} \sim 1 \mathrm{~d}$ 的结果相吻合，但石墨态碳的平均晶化尺寸 无显著变化. 在 $\mathrm{Ar}$ 气氛中, 对 $700{ }^{\circ} \mathrm{C}$ 制得的 $\mathrm{hCNCs}$ 分 别在 900、1100 和 $1300{ }^{\circ} \mathrm{C}$ 下退火处理 $4 \mathrm{~h}$, 样品分别记

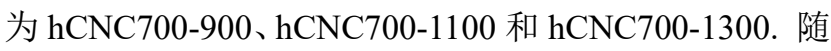
退火温度升高, $I_{\mathrm{D}} / I_{\mathrm{G}}$ 从 3.0 下降到 2.7 和 $2.0, \mathrm{FWHM}(\mathrm{D})$ 从 $254 \mathrm{~cm}^{-1}$ 下降到 219 和 $144 \mathrm{~cm}^{-1}$, 而 $\mathrm{FWHM}(\mathrm{G})$ 保持 在约 $110 \mathrm{~cm}^{-1}$ (图 $2 \mathrm{~d}, 2 \mathrm{e}$ ). 说明随退火温度升高, 碳纳米 笼的缺陷显著减少，但石墨态碳的平均晶化尺寸变化不 大. 因此, 升高制备和退火温度可有效提高 hCNCs 的石 墨化程度, 减少缺陷数量.

$\mathrm{hCNCs}$ 的氮气吸附-脱附曲线呈现典型的 IV 型特 征, 存在两个相互连通的迟滞环, 结合孔分布曲线可知, $\mathrm{hCNCs}$ 具有微孔-介孔-大孔共存的分级孔结构(图 S1) ${ }^{[37]}$. hCNC700、hCNC800 和 hCNC900 的比表面积依 次为 $2300 、 1810$ 和 $1400 \mathrm{~m}^{2} \cdot \mathrm{g}^{-1}$ (表 S1). 如此高的比表 面积可以提供丰富的催化活性位点, 相互连通的分级孔 结构有利于物料的传输, 使反应物与活性位点充分接 

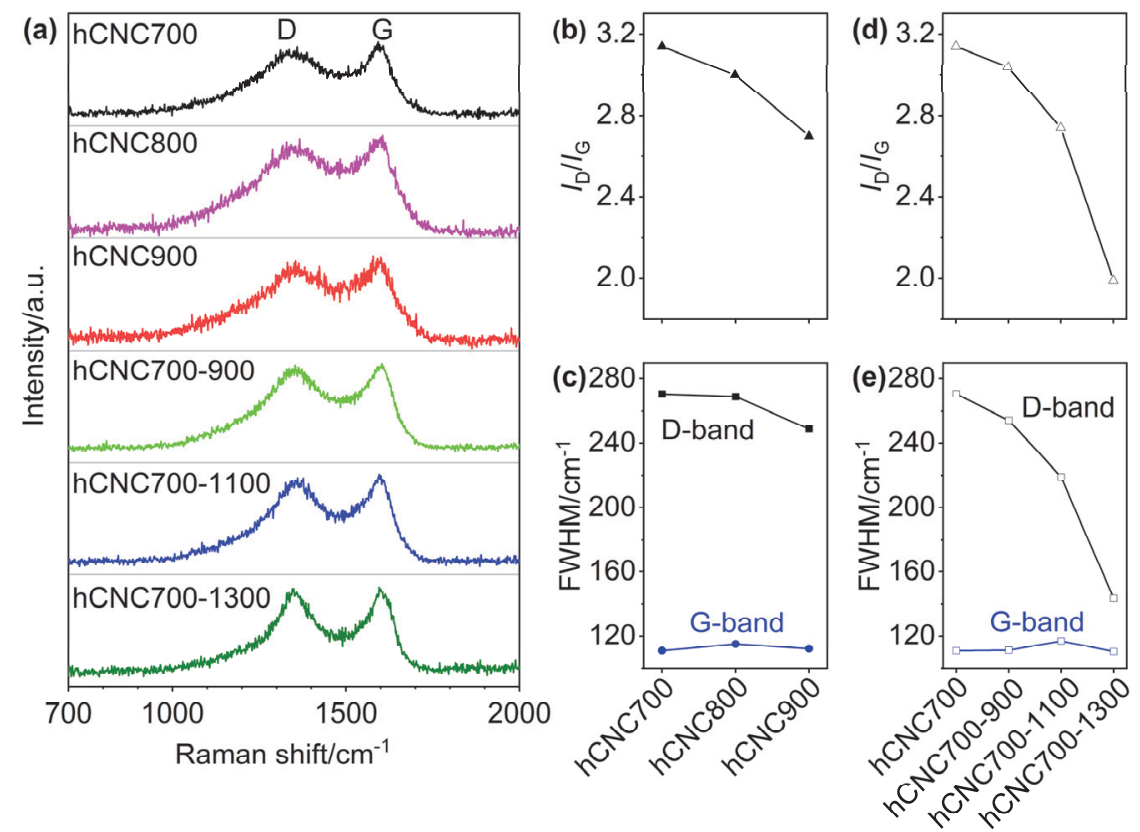

图 $2 \mathrm{hCNCs}$ 系列样品的 Raman 表征结果. (a) Raman 光谱; (b, d) $I_{\mathrm{D}} / I_{\mathrm{G}}$ 随 $\mathrm{hCNCs}$ 制备温度(b)和退火温度(d)的变化关系; (c, e) D 带和 G 带半峰宽 随 hCNCs 制备温度(c)和退火温度(e)的变化关系.

Figure 2 Raman characterizations of hCNCs. (a) Raman spectra; (b, d) $I_{\mathrm{D}} / I_{\mathrm{G}}$ versus synthesis and annealing temperatures of hCNCs, respectively; (c, e) FWHMs of D- and G-band versus synthesis and annealing temperatures of hCNCs, respectively.

触. X 射线光电子能谱(XPS)除 C 信号外仅有微弱的 $\mathrm{O}$ 信号, 没有 $\mathrm{Mg}$ 等金属的信号(图 S2), 且 $\mathrm{X}$ 射线衍射 (XRD)结果中未见 $\mathrm{MgO}$ 衍射峰(图 S3), 表明 $\mathrm{MgO}$ 模板 已被除尽, $\mathrm{hCNCs}$ 中不含金属杂质.

以 $\mathrm{hCNC} 700$ 在无溶剂条件下催化苄胺氧化偶联反 应(温度: $100{ }^{\circ} \mathrm{C}$; $\mathrm{O}_{2}$ 流量: $20 \mathrm{~mL} \cdot \mathrm{min}^{-1}$; 反应时间: $8 \mathrm{~h}$ ), 并与还原氧化石墨烯 $(\mathrm{rGO})$ 、碳纳米管 $(\mathrm{CNT}) 、 \mathrm{XC}-72$ 及 活性炭(AC)等典型碳基无金属催化剂进行对比. 表 1 列 出这些碳材料的比表面积、用量及相应的苄胺转化率和 $N$-茮烯丁胺选择性. 在不加催化剂的空白实验中, 茮胺 转化率为 0 , 说明在此实验条件下茮胺不能被氧气直接 氧化. hCNC700 的芐胺转化率为 $98 \%, N$-芐烯丁胺的选 择性 $>98 \%$. 在保持催化剂的总表面积相近的情况下, $\mathrm{rGO} 、 \mathrm{CNT} 、 \mathrm{XC}-72$ 和 $\mathrm{AC}$ 的 $N$-芐烯丁胺选择性均 $>95 \%$, 但苄胺转化率分别为 $70 \% 、 22 \% 、 20 \%$ 和 $8 \%$, 均大大低 于 $\mathrm{hCNC} 700$ 的 $98 \%$. 图 3 是 hCNCs 系列催化剂的苄胺 氧化偶联催化性能对比. 将单位质量的碳基催化剂在单 位时间内转化的苄胺的物质的量定义为转换频率 $(\mathrm{TOF})^{[19]}$. hCNC700 的表观 TOF 显著优于几种典型碳材 料和文献报道的碳基催化剂(图 3a) ${ }^{[17-20]}$. 将 $\mathrm{hCNC} 700$ 从催化反应系统中分离、回收, 其形貌和结构无明显变 化(图 S4), 循环使用 6 次后, 反应 $8 \mathrm{~h}$ 的苄胺转化率依然 保持在 $96 \%$ 以上(图 3b), 表明 $\mathrm{hCNC} 700$ 具有优秀的循环 稳定性. 可见, hCNC700 是一种优异的苄胺氧化偶联制 $N$-茮烯丁胺催化剂.
表 $1 \mathrm{hCNC700}$ 与其他典型碳基无金属催化剂对芐胺氧化偶联制 $N$ 苄烯丁胺的催化性能对比 ${ }^{a}$

Table 1 Comparison of catalytic performance of hCNC700 and other typical carbon-based metal-free catalysts for the oxidative coupling of benzylamine to $N$-benzylidene benzylamine

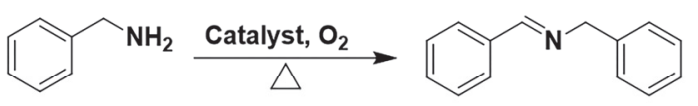
benzylamine

$N$-benzylidene benzylamine

\begin{tabular}{cccccc}
\hline Catalyst & $\begin{array}{c}\text { Conversion/ } \\
\%\end{array}$ & $\begin{array}{c}\text { Selectivity/ } \\
\%\end{array}$ & $\begin{array}{c}S_{\mathrm{BET}^{b} /} \\
\left(\mathrm{m}^{2} \bullet \mathrm{g}^{-1}\right)\end{array}$ & $\begin{array}{c}S_{\text {total }}{ }^{c} / \\
\mathrm{m}^{2}\end{array}$ & $\begin{array}{c}\text { Mass/ } \\
\mathrm{mg}\end{array}$ \\
\hline Blank & 0 & - & - & - & - \\
hCNC700 & 98 & $>98$ & 2300 & 46.0 & 20.0 \\
rGO & 70 & $>95$ & 2000 & 40.0 & 20.0 \\
CNT & 22 & $>98$ & 230 & 46.0 & 200 \\
XC-72 & 20 & $>98$ & 300 & 42.0 & 140 \\
AC & 8 & $>98$ & 150 & 42.0 & 280 \\
\hline
\end{tabular}

${ }^{a}$ Reaction conditions: $5.0 \mathrm{~g}$ benzylamine, $20 \mathrm{~mL} \cdot \min ^{-1} \mathrm{O}_{2}, 100{ }^{\circ} \mathrm{C}, 8 \mathrm{~h}$, using carbon catalysts with similar surface area; ${ }^{b}$ specific surface area; ${ }^{c}$ surface area of the used carbon material.

在相同反应条件下，随温度升高制得的 hCNC700、 $\mathrm{hCNC} 800$ 和 $\mathrm{hCNC} 900$ 样品的芐胺转化率依次为 $98 \%$ 、 $81 \%$ 和 73\%(图 3c). 该变化趋势与 $\mathrm{hCNCs}$ 的比表面积下 降(表 S1)、表面缺陷减少(图 $2 b, 2 c$ ) 的变化趋势一致, 因 此, 芐胺氧化的催化活性很可能来源于 hCNCs 的表面 缺陷.

为了验证上述推定，我们将制备 $\mathrm{hCNCs}$ 的温度固 定在 $700{ }^{\circ} \mathrm{C}$, 增加碳源(苯)的用量, 使纳米笼壁变厚, 

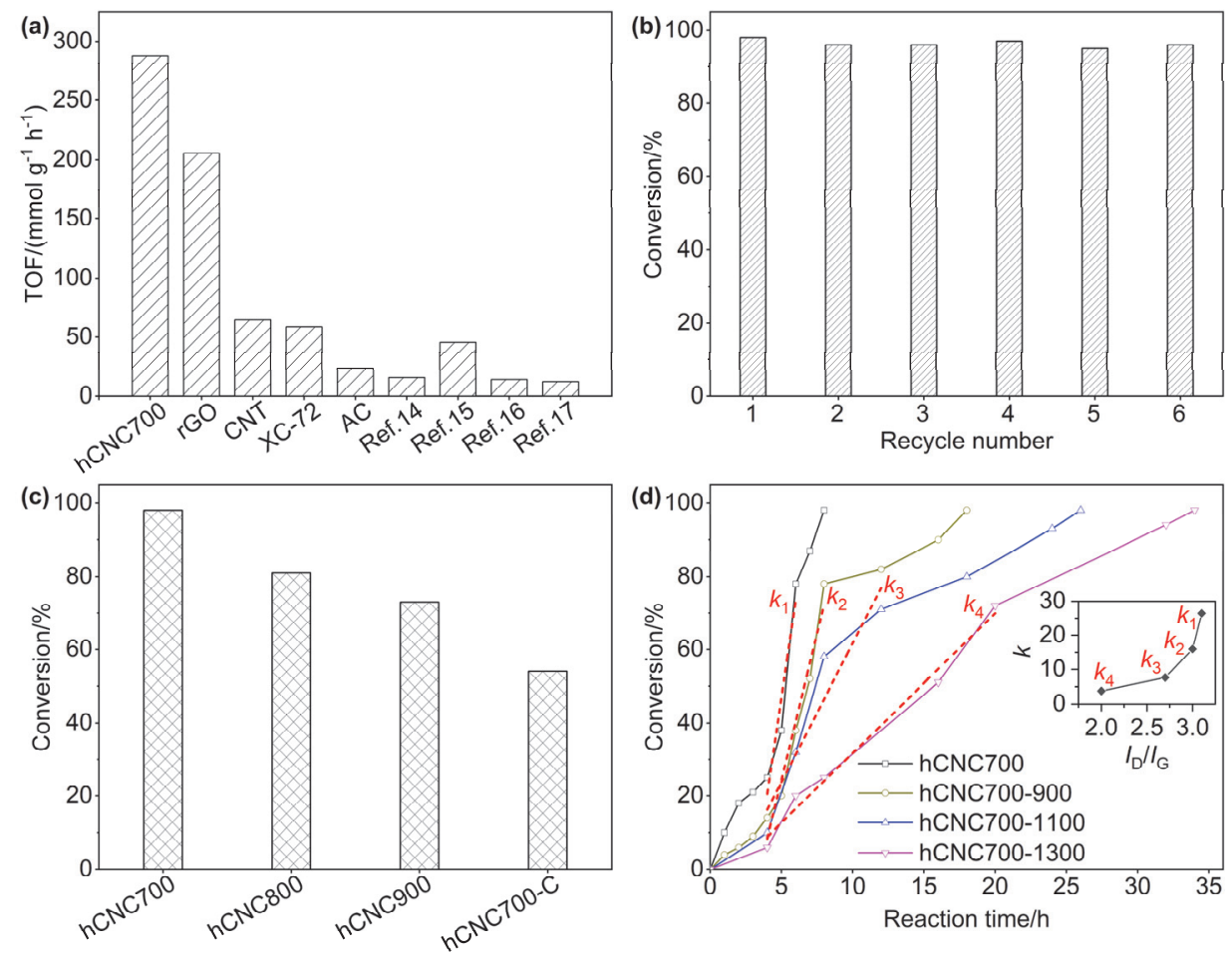

图 $3 \mathrm{hCNCs}$ 系列催化剂的芐胺氧化偶联催化性能对比. (a) hCNC700 与几种典型碳材料(rGO、CNT、XC-72 及 AC)的苄胺转换频率(TOF)对比. 文 献[14-17]碳基无金属催化剂的相应数据供参考; (b) hCNC700 的循环稳定性; (c) hCNC700、hCNC800、hCNC900 和 hCNC700-C 反应 $8 \mathrm{~h}$ 后的转化 率; (d) hCNC700 及经不同温度退火后的 $\mathrm{hCNC} 700$ 的转化率随时间变化曲线. 插图为对快速反应阶段进行线性拟合所得斜率 $k$ 值与 $I_{\mathrm{D}} / I_{\mathrm{G}}$ 的关系.

Figure 3 Comparison of catalytic performance of hCNCs for the oxidative coupling of benzylamine. (a) Comparison of the turnover frequency (TOF) of benzylamine for hCNC700 and several typical carbon-based materials (rGO, CNT, XC-72 and AC). The data for the carbon-based metal-free catalysts from Refs [14-17] are also listed for reference; (b) cycling stability of hCNC700; (c) benzylamine conversions of hCNC700, hCNC800, hCNC900 and $\mathrm{hCNC} 700-\mathrm{C}$ after reacting for $8 \mathrm{~h}$; (d) benzylamine conversions of the as-prepared hCNC700 and the annealed hCNC700 at different temperatures versus time. Inset is the curve of $k$ versus $I_{\mathrm{D}} / I_{\mathrm{G}}$, here $k$ is the linearly-fitted slope in the fast reaction period marked in (d).

所得样品的比表面积减小为 $900 \mathrm{~m}^{2} \cdot \mathrm{g}^{-1}$ (命名为 hCNC700-L), 单位质量的表面缺陷数量也相应下降. 在相同的反应条件下, 反应 $8 \mathrm{~h}$ 后苄胺的转化率从 $\mathrm{hCNC} 700$ 的 $98 \%$ 大幅降至 hCNC700-L 的 $9.0 \%$, 反应 18 $\mathrm{h}$ 后才达到 90\%(图 S5). 为了排除比表面积的影响, 在 $\mathrm{Ar}$ 气氛下高温退火处理 hCNC700, 在保持比表面积相 近 $\left(2300 \sim 2050 \mathrm{~m}^{2} \cdot \mathrm{g}^{-1}\right)$ 的情况下提高其石墨化程度, 减 少表面缺陷(图 2, 表 S1), 茮胺氧化的速率随之显著下 降(图 3d): (i)在 $8 \mathrm{~h}$ 时, hCNC700、hCNC700-900、 hCNC700-1100 和 hCNC700-1300 的苄胺转化率分别为 $98 \% 、 78 \%$ 、58\%和 25\%; (ii)达到 98\%的苄胺转化率所 需反应时间依次为 $8 、 18 、 26$ 和 $34 \mathrm{~h}$; (iii)对快速反应阶 段进行线性拟合, 其斜率 $k$ 与催化剂的本征活性密切相 关, 所得 $k$ 值随 $I_{\mathrm{D}} / I_{\mathrm{G}}$ 的减小而减小. 这些结果进一步证 实 hCNCs 的表面缺陷是苄胺氧化偶联的催化活性位点. 进一步分析可知, 退火处理不会改变碳纳米笼的形貌和 尺寸, 但能部分消除边缘缺陷, 相比于 hCNC700 样品, hCNC700-900、hCNC700-1100 和 hCNC700-1300 的边 缘缺陷逐渐减少, 催化性能逐渐下降. 这表明 $\mathrm{hCNCs}$ 的 边缘缺陷是其主要催化活性位点.

文献中通常将碳基无金属催化剂催化伯胺氧化偶
联的活性归因于杂原子掺杂 [19]、羧酸官能团与边缘缺陷 的协同作用 ${ }^{[17]}$ 和碳材料的本征缺陷 ${ }^{[18]}$. 我们的 hCNCs 催化剂未经掺杂或羧酸官能团修饰, 说明碳的本征缺陷 就具有十分优异的茮胺氧化偶联催化活性, 这是因为本 征缺陷破坏了 $\pi$ 共轭体系的完整性，活化了 $\pi$ 电子，从 而表现出催化活性 ${ }^{[18,23]}$.

上述结果表明, hCNC700 具有十分优异的苄胺氧化 偶联催化性能，表面缺陷是该催化反应的活性位点. hCNC700 超高的比表面积提供了大量的缺陷活性位点, 而其独特的分级孔结构有利于反应过程中的传质，使这 些活性位点得以充分利用. 为了验证分级孔结构的作 用，我们利用毛细作用破坏了 $700{ }^{\circ} \mathrm{C}$ 下制得的薄壁 $\mathrm{hCNCs}$ 的分级孔结构(hCNC700-C) $)^{[38]}$. 该催化剂 $8 \mathrm{~h}$ 的 芐胺转化率仅为 $54 \%$, 远低于 hCNC700 的 98\%(图 3c), 说明了分级孔结构的重要作用.

进一步，我们考察了 $\mathrm{hCNC700}$ 对含不同取代基的 亚甲基胺化合物的氧化偶联反应的催化性能, 结果见表 2. 在相同条件下反应 $12 \mathrm{~h}$, 所有氧化偶联反应产物(亚 胺)的选择性均超过 $95 \%$, 但芳香族取代基化合物的转 化率显著高于脂环族和脂肪族取代基的化合物，前者的 转化率均超过 $90 \%$, 而后两者的转化率仅为 $40 \%$ 和 
$15 \%$. 这可能是由于芳香族取代基与 hCNC700 的 $\mathrm{sp}^{2}$ 碳 之间存在较强的 $\pi-\pi$ 相互作用 ${ }^{[39]}$, 有利于其在 hCNC700 表面的吸附，从而促进反应. 由上述结果可知， hCNC700 对芳香族亚甲基胺化合物具有优异的催化性 能，即具有优秀的底物拓展性.

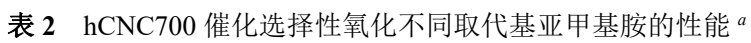

Table 2 Catalytic performance of selective oxidation of substituted methylene amine with hCNC700

底物

${ }^{a}$ Reaction conditions: $5.0 \mathrm{~g}$ methylene amine compounds, $20 \mathrm{mg}$ hCNC700, $20 \mathrm{~mL} \cdot \min ^{-1} \mathrm{O}_{2}, 100{ }^{\circ} \mathrm{C}, 12 \mathrm{~h}$.

\section{3 结论}

我们发展了一种性能优异的碳基无金属苄胺氧化 偶联催化剂——分级孔结构碳纳米笼, 在无溶剂、 $100{ }^{\circ} \mathrm{C}$ 和常压 $\mathrm{O}_{2}$ 条件下即可实现 $N$-茮烯丁胺的高效合 成，表面缺陷是该催化反应的活性位点. 反应 $8 \mathrm{~h}$ 后， hCNC700 的茮胺转化率可达 98\%, 远优于 rGO、CNT、 XC-72 及 $\mathrm{AC}$ 等典型碳材料; $N$-芐烯丁胺选择性可达 98\%; 循环使用 6 次后催化性能基本无衰减; 其表观 TOF 显著优于几种典型碳材料和文献报道的碳基无金 属催化剂. 并且, 该催化剂具有优异的底物拓展性, 对 芳香族亚甲基胺化合物的氧化偶联均有高催化活性和 高选择性. hCNC700 的优异催化性能源于其独特的分级 孔结构有利于反应过程中的传质, 使丰富的表面活性位 点(缺陷)得以充分利用, 而超高的比表面积可提供大量 的缺陷活性位点. 本研究为开发廉价高效的碳基无金属 催化剂提供了新的思路.

\section{4 实验部分}

\section{1 催化剂制备}

hCNCs 的制备: 采用我们课题组之前报道的制备 方法 ${ }^{[37]}$. 称取 $4 \mathrm{~g}$ 的碱式碳酸镁 $\left(4 \mathrm{MgCO}_{3} \bullet \mathrm{Mg}(\mathrm{OH})_{2} \bullet\right.$
$5 \mathrm{H}_{2} \mathrm{O}$ ) 置于石英管中, 在 $100 \mathrm{~mL} \cdot \mathrm{min}^{-1}$ 的 $\mathrm{Ar}$ 气流保护下 将管式炉以 $10{ }^{\circ} \mathrm{C} \cdot \mathrm{min}^{-1}$ 升至指定温度 $(700 、 800$ 和 $900{ }^{\circ} \mathrm{C}$ ), 用平流洜以 $66 \mu \mathrm{L} \cdot \mathrm{min}^{-1}$ 的流量通入碳源 (苯) $0.5 \mathrm{~mL}$ ，进样过程中每间隔 $1 \mathrm{~min}$ 用大气流冲洗 $5 \mathrm{~s}$, 保温 $2 \mathrm{~h}$ 后自然冷却. 将产物放入 $6 \mathrm{~mol} \cdot \mathrm{L}^{-1}$ 盐酸中摚拌 $24 \mathrm{~h}$ 去除 $\mathrm{MgO}$ 模板, 过滤, 用去离子水洗净, $80{ }^{\circ} \mathrm{C}$ 真空 干燥 $12 \mathrm{~h}$, 即得 hCNCs. 按照制备温度分别记为

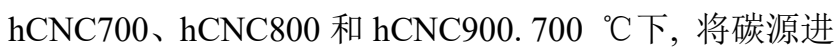
样量减少到 $0.2 \mathrm{~mL}$ 制得的塌缩的样品命名为 hCNC700- $\mathrm{C}^{[38]}$; 将碳源进样量增加到 $1.5 \mathrm{~mL}$ 制得的低 比表面积的样品命名为 hCNC700-L.

hCNC700 的退火处理：将 hCNC700 置于管式炉中, 在 $100 \mathrm{~mL} \cdot \mathrm{min}^{-1}$ 的 $\mathrm{Ar}$ 气氛下以 $10{ }^{\circ} \mathrm{C} \cdot \mathrm{min}^{-1}$ 升至指定 温度 $\left(t=900 、 1100 、 1300{ }^{\circ} \mathrm{C}\right)$, 保温 $4 \mathrm{~h}$, 自然冷却至室

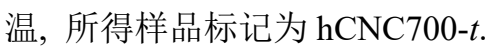

\section{2 催化剂形貌结构和成分表征}

采用高分辨透射电子显微镜(HRTEM, JEM-2100F, $200 \mathrm{kV}$ )、扫描电子显微镜(SEM, Hitachi S4800, $20 \mathrm{kV}$ )、 $X$ 射线衍射(XRD, Bruker, D8 Advance A25)、X 射线光 电子能谱(XPS, PHI 5000 VersaProbe)和 Raman 光谱 (Horiba Scientific, $514 \mathrm{~nm}$ )表征催化剂的形貌、结构和成 分. 氮气等温吸脱附测试 $(77 \mathrm{~K})$ 是在 Thermo Fisher Scientific Surfer Gas Adsorption Porosimeter 上完成的, 实验 时先将样品在 $0.13 \mathrm{~Pa} 、 300{ }^{\circ} \mathrm{C}$ 条件下预处理 $6 \mathrm{~h}$. 采用 Brunauer-Emmett-Teller (BET)、Horvath-Kawazoe (HK, $<2 \mathrm{~nm}$ ) 和 Barrett-Joyner-Halenda (BJH，>2 nm) 方法处 理吸附支的数据得到比表面积、微孔和介孔-大孔分布.

\section{3 催化反应及性能表征}

茮胺氧化偶联反应: 在无溶剂条件下, 将 $5 \mathrm{~g}$ 芐胺、 $20 \mathrm{mg}$ 催化剂加入 $20 \mathrm{~mL}$ 耐压玻璃管中, $100{ }^{\circ} \mathrm{C}$ 加热回 流, 通入 $20 \mathrm{~mL} \cdot \mathrm{min}^{-1}$ 的 $\mathrm{O}_{2}$ (鼓泡)并摚拌. 取 $0.10 \mathrm{~mL}$ 反 应液(用 $0.22 \mu \mathrm{m}$ 聚四氟乙烯滤膜过滤), 加入 $0.50 \mathrm{~mL}$ 乙 腈、 $20 \mu \mathrm{L}$ 均三甲苯外标物, 采用气相色谱-质谱联用 (GCMS-QP2010)做定性分析，气相色谱 GC-7900(色谱 柱: HP-5)做定量分析(标准曲线见图 S6). 将催化剂离心 分离, 用乙醇洗涤 3 次, $200{ }^{\circ} \mathrm{C}$ 真空干燥 $12 \mathrm{~h}$, 再在相同 条件下进行循环催化实验.

\section{References}

[1] Chen, B.; Wang, L.; Gao, S. ACS Catal. 2015, 5, 5851.

[2] Qin, J.; Long, Y.; Wu, W.; Zhang, W.; Gao, Z.; Ma, J. J. Catal. 2019, $371,161$.

[3] Cao, X.; Qin, J.; Gou, G.; Li, J.; Wu, W.; Luo, S.; Luo, Y.; Dong, Z.; Ma, J.; Long, Y. Appl. Catal. B Environ. 2020, 272, 118958.

[4] Reeves, J. T.; Visco, M. D.; Marsini, M. A.; Grinberg, N.; Busacca, C. A.; Mattson, A. E.; Senanayake, C. H. Org. Lett. 2015, 17, 2442.

[5] Furukawa, S.; Suga, A.; Komatsu, T. ACS Catal. 2015, 5, 1214.

[6] He, W.; Wang, L.; Sun, C.; Wu, K.; He, S.; Chen, J.; Wu, P.; Yu, Z. Chem. Eur. J. 2011, 17, 13308.

[7] Mielby, J.; Poreddy, R.; Engelbrekt, C.; Kegnæs, S. Chin. J. Catal. 2014, 35, 670 . 
[8] Zhang, L. L.; Wang, W. T.; Wang, A. Q.; Cui, Y. T.; Yang, X. F.; Huang, Y. Q.; Liu, X. Y.; Liu, W. G.; Son, J. Y.; Oji, H.; Zhang, T. Green Chem. 2013, 15, 2680.

[9] Gong, K. P.; Du, F.; Xia, Z. H.; Durstock, M.; Dai, L. M. Science 2009, 323, 760.

[10] Yang, L. J.; Shui, J. L.; Du, L.; Shao, Y. Y.; Liu, J.; Dai, L. M.; Hu, Z. Adv. Mater. 2019, 31, 1804799.

[11] Zheng, Y.; Jiao, Y.; Zhu, Y.; Li, L. H.; Han, Y.; Chen, Y.; Du, A.; Jaroniec, M.; Qiao, S. Z. Nat. Commun. 2014, 5, 3783.

[12] Jiao, Y.; Zheng, Y.; Davey, K.; Qiao, S. Z. Nat. Energy 2016, 1, 16130.

[13] Zhang, J.; Liu, X.; Blume, R.; Zhang, A.; Schlögl, R.; Su, D. S. Science 2008, 322, 73.

[14] Yu, H.; Peng, F.; Tan, J.; Hu, X.; Wang, H.; Yang, J.; Zheng, W. Angew. Chem. Int. Ed. 2011, 50, 3978.

[15] Wen, G.; Wu, S.; Li, B.; Dai, C.; Su, D. S. Angew. Chem. Int. Ed. 2015, 54, 4105 .

[16] Yang, S. L.; Peng, L.; Huang, P. P.; Wang, X. S.; Sun, Y. B.; Cao, C. Y.; Song, W. G. Angew. Chem. Int. Ed. 2016, 55, 4016.

[17] Su, C. L.; Acik, M.; Takai, K.; Lu, J.; Hao, S. J.; Zheng, Y.; Wu, P. P.; Bao, Q. L.; Enoki, T.; Chabal, Y. J.; Loh, K. P. Nat. Commun. 2012, 3, 9 .

[18] Chen, B.; Wang, L. Y.; Dai, W.; Shang, S. S.; Lv, Y.; Gao, S. ACS Catal. 2015, 5, 2788.

[19] Wang, K. Z.; Jiang, P. B.; Yang, M.; Ma, P.; Qin, J. H.; Huang, X. K.; Ma, L.; Li, R. Green Chem. 2019, 21, 2448.

[20] He, H. W.; Li, Z.; Li, K.; Lei, G. Y.; Guan, X. L.; Zhang, G. L.; Zhang, F. B.; Fang, X. B.; Peng, W. C.; Li, Y. ACS Appl. Mater. Interfaces 2019, 11, 31844 .

[21] Yang, L. J.; Jiang, S. J.; Zhao, Y.; Zhu, L.; Chen, S.; Wang, X. Z.; Wu, Q.; Ma, J.; Ma, Y. W.; Hu, Z. Angew. Chem. Int. Ed. 2011, 50, 7132 .

[22] Zhao, Y.; Yang, L. J.; Chen, S.; Wang, X. Z.; Ma, Y. W.; Wu, Q.; Jiang, Y. F.; Qian, W. J.; Hu, Z. J. Am. Chem. Soc. 2013, 135, 1201.

[23] Jiang, Y. F.; Yang, L. J.; Sun, T.; Zhao, J.; Lyu, Z. Y.; Zhuo, O.; Wang, X. Z.; Wu, Q.; Ma, J.; Hu, Z. ACS Catal. 2015, 5, 6707.
[24] Zhong, G. Y.; Wang, H. J.; Yu, H.; Peng, F. Acta Chim. Sinica 2017, 75,943 (in Chinese). (钟国玉, 王红娟, 余皓, 彭峰, 化学学报, 2017, 75, 943.)

[25] Li, W.; Wang, D.; Zhang, Y.; Tao, L.; Wang, T.; Zou, Y.; Wang, Y.; Chen, R.; Wang, S. Adv. Mater. 2020, 32, 1907879.

[26] Wu, Q.; Yang, L. J; Wang, X. Z.; Hu, Z. Acc. Chem. Res. 2017, 50, 435.

[27] Zhang, Z. Q.; Ge, C. X.; Chen, Y. G.; Wu, Q.; Yang, L. J.; Wang, X Z.; Hu, Z. Acta Chim. Sinica 2019, 77, 60 (in Chinese). (张志琦, 葛 承宣, 陈玉刚, 吴强, 杨立军, 王喜章, 胡征, 化学学报, 2019, 77, 60.)

[28] Wu, Q.; Yang, L. J; Wang, X. Z.; Hu, Z. Adv. Mater. 2020, 32, 1904177.

[29] Wu, Q.; Yang, L. J.; Wang, X. Z.; Hu, Z. Sci. China Chem. 2020, 63, 665.

[30] Tang, G. A.; Mao, K.; Zhang, J.; Lyu, P.; Cheng, X. Y.; Wu, Q.; Yang, L. J.; Wang, X. Z.; Hu, Z. Acta Chim. Sinica 2020, 78, 444 (in Chinese). (汤功奥, 毛鲲, 张静, 吕品, 程雪怡, 吴强, 杨立军, 王喜章, 胡征, 化学学报, 2020, 78, 444.)

[31] Zhang, J.; Tang, G. A.; Zeng, Y.; Wang, B. X.; Liu, L. W.; Wu, Q.; Yang, L. J.; Wang, X. Z.; Hu, Z. Acta Chim. Sinica 2020, 78, 572 (in Chinese). (张静, 汤功奥, 曾誉, 王保兴, 刘力玮, 吴强, 杨立军, 王喜章, 胡征, 化学学报, 2020, 78, 572.)

[32] Ferrari, A. C.; Robertson, J. Phys. Rev. B 2000, 61, 14095.

[33] Dresselhaus, M. S.; Jorio, A.; Hofmann, M.; Dresselhaus, G.; Saito, R. Nano Lett. 2010, 10, 751.

[34] Ferrari, A. C.; Basko, D. M. Nat. Nanotechnol. 2013, 8, 235.

[35] Bourrat, X.; Langlais, F.; Chollon, G.; Vignoles, G. L. J. Braz. Chem. Soc. 2006, 17, 1090.

[36] Maslova, O. A.; Ammar, M. R.; Guimbretière, G.; Rouzaud, J. N.; Simon, P. Phys. Rev. B 2012, 86, 134205.

[37] Xie, K.; Qin, X. T.; Wang, X. Z.; Wang, Y. N.; Tao, H. S.; Wu, Q.; Yang, L. J.; Hu, Z. Adv. Mater. 2012, 24, 347.

[38] Bu, Y. F.; Sun, T.; Cai, Y. J.; Du, L. Y.; Zhuo, O.; Yang, L. J.; Wu, Q.; Wang, X. Z.; Hu, Z. Adv. Mater. 2017, 29, 1700470.

[39] Grimme, S. Angew. Chem. Int. Ed. 2008, 47, 3430.

(Cheng, B.) 